\title{
Evaluation of agarose gel electrophoresis for characterization of silver nanoparticles in
}

industrial products

Maria S. Jimenez*, Jose M. Luque-Alled, Teresa Gomez, Juan R. Castillo

Environmental Sciences Institute (IUCA), Analytical Spectroscopy and Sensors Group (GEAS),

University of Zaragoza, Pedro Cerbuna, 12, Zaragoza, 50009, Spain

\section{List of Abbrevations}

Agarose Gel Electrophoresis

Bis(p-sulfonatophenyl)phenylphosphine

BP

Electrophoresis buffer

EB

1-ethyl-3-(-dimethylaminopropyl) carbodiimide hydrochloride $\quad$ EDC

Field Flow Fractionation

FFF

Gel Electrophoresis

GE

Hydrodynamic Chromatography

HDC

Laser Ablation

LA

Loading buffer

LB

Mercaptosuccinic acid

TMA

Micro RNA

miRNA

Molecular weight

MW

Nanomaterials

NMs

Nanoparticles

NPs

Protein corona

PC

Quantum dots

QDs

Sodium dodecylbenzenesulfonate

SDBS

Sulphate-stabilized polystyrene

SSPS

Standards

Sts

Size to charge ratio

$\mathrm{s} / \mathrm{z}$

Transmission electron microscopy

TEM

\footnotetext{
*To whom correspondence should be addressed: (Phone: 34 976762257, email: jimenezm@unizar.es)
} 
Keywords: Agarose gel electrophoresis, Characterization, Laser-Ablation-ICP-MS, Protein corona, Silver Nanoparticles.

Total number or words: 5092 (including references as well as table and figure legends; list of abbreviations, keywords and abstract are not included). 


\begin{abstract}
Agarose gel electrophoresis (AGE) has been used extensively for characterization of pure nanomaterials or mixtures of pure nanomaterials. We have evaluated the use of AGE for characterization of Ag nanoparticles (NPs) in an industrial product (described as strong antiseptic). Influence of different stabilizing agents (PEG, SDS and Sodium dodecylbenzenesulfonate), buffers (TBE and Tris Glycine) and functionalizing agents (Mercaptosuccinic acid (TMA) and proteins) have been investigated for the characterization of AgNPs in the industrial product using different sizes-AgNPs standards. The use of $1 \%$ SDS, $0.1 \%$ TMA and Tris Glycine in gel, electrophoresis buffer and loading buffer led to the different sizes-AgNPs commercial standards moved according to their size/charge ratio (obtaining a linear relationship between apparent mobility and mean diameter). After using SDS and TMA, the behaviour of the AgNPs in the industrial product (containing a casein matrix) was completely different, being not possible their size characterization. However we demonstrated that AGE with LA-ICP-MS detection is an alternative method to confirm the protein corona formation between the industrial product and two proteins (Bovine Serum Albumin and transferrin) maintaining NPs-protein binding (what is not possible using SDS-PAGE).
\end{abstract}




\section{Introduction}

The last years witnessed a blooming of nanotechnology and the use of nanoparticles (NPs) and nanomaterials (NMs) for scientific purpose and commercial applications is continuously expanding. Different properties of NMs such as size, shape, structure, chemical composition, concentration, agglomeration state, surface charge, surface functionality and porosity play a major role that characterize NMs and their environmental and health impact. Samples subjected to the analysis are often composed of populations of polydispersed nanoparticles. If separation of subpopulations of nanoparticles present in the sample is required, electrophoretic techniques should be taken into account. Electrophoretic methods for nanoanalysis can provide inexpensive and efficient tools for quality assurance and safety control. Actually different reviews have been published regarding to electrophoretic methods for separation, identification and characterization of several NPs [1-3], gold [4] and silver NPs [5].

Gel electrophoresis (GE) is a separation technique based on the different migration behaviour of analytes in gel by sieve effects under electric field. Compared to other separation techniques such as centrifugation, HPLC, SEC, Field Flow Fractionation (FFF), Hydrodynamic Chromatography (HDC), CE, GE has the advantage of being a simple and economic method, allowing multiple runs in parallel on the same gel [6]. The most commonly used GE methods include PAGE [7], commonly used to separate protein molecules to a high degree of purity, and agarose GE (AGE) mainly used for separating charged biopolymers, such as DNA and RNA [8]. Although PAGE has been used for characterization of NPs, such as CdTe Quantum dots (QDs) bioconjugates [9] and CdTe QDs stabilized with mercaptosuccinic acid [10], the small pore size of polyacrylamide gels (usually of less than $10 \mathrm{~nm}$ ) limits its application for other NPs. By contrast, the larger pore size of agarose gels $(10-100 \mathrm{~nm})$ enables the wide applications of AGE in NPs fractionation. Although AGE has been previously used to characterize a variety of NPs under certain conditions [1-5] the main applications have been focused on characterizing pure NMs or mixtures of pure NMs and not on the characterization of NPs in industrial or consumer products (until our knowledge). For example separation of a mixture of gold nanosphere and nanoplates with a wide SD (21\% for the former and 30\% for the latter) synthesized in the same 
batch was achieved via density gradient centrifugation and the subsequent shape separation via AGE [11].

In most references, to stabilize the NPs, they have been functionalized after or during the synthesis previously to be characterized by AGE. One of the salient features of AGE is that it can be used to confirm if the functionalization has been successful. In one of the most cited paper regarding to the characterization of NPs by AGE, Hanauer et al. [6] demonstrated the separation of gold and silver NPs according to their size and shape in $0.2 \%$ agarose gels after NPs coating with PEG. They analyzed the particle distribution in the various regions of the gel by two methods: local extinction spectroscopy and transmission electron microscopy (TEM). The coupling of biomolecules to NPs is quite challenging because of the increasing applications of nanotechnology in biomedical and physical sciences. Several reports demonstrate the successful coupling of biomolecules to water-soluble NPs using EDC [1-ethyl-3-(dimethylaminopropyl) carbodiimide hydrochloride] or its derivatives as the main coupling reagent. Bartczak et al. [12] used AGE for the characterization of conjugates obtained after optimization of peptide-functionalized gold NPs using EDC/Sulfo-NHS coupling. The successful coupling for the different reaction parameters, was evaluated using $0.75 \%$ agarose gel and 0.5xTBE buffer. More recently, Beskorovaynyy et al. [13] presented a high-throughput two dimensional-grid GE cell based method capable of optimizing the $\mathrm{pH}$ and concentration of different thiol-containing acidic agents used for surface modification of gold nanorods. The system was able to optimize the surface modification of 96 samples of gold NPs in the same run in approximately $1 \mathrm{~h}$.

The effective delivery of RNA and DNA into appropriate cells for genetic manipulation or cellular marking without significant cytotoxicity remains challenging. MicroRNA (miRNA) conjugated gold NPs have been used as delivery vehicle because of their unique optical properties, low cytotoxicity and enhanced lifespan in the bloodstream [14]. Assessment of the conjugation and stability of the NPs with miRNA was performed using AGE testing the highly charged nature of the miRNA on the AuNPs. It was observed that the unmodified particles (with citrate capping) basically stayed in the well of the gel due to the loss of weakly bound 
citrate ligands, whereas the bis(p-sulfonatophenyl)phenylphosphine)-capped particles travelled the farthest in the gel as a result of the high level of charges on the particles.

As described above for citrate-capped AuNPs (which basically stayed in the well of the gel due to the due to the loss of weakly bound citrate ligands) it is important to highlight that simply introducing anionic NPs in standard large-pore agarose gels does not automatically ensure propagation or size-separation because attractive interactions can exist between the gel and the NPs. Whereas many kinds of highly charged biopolymers, such as DNA, do not bind to common agarose gels at typical conditions in standard buffers, NPs, which have a much larger size and a comparatively smaller overall charge density, could bind strongly to the gel. Whereas altering the surface of the NPs is a possible solution, if characterization of NPs in a real sample is looked for, the use of agarose gels with a stabilizing agent could be a better solution. Zhu et al. [15] studied the use of PEG and SDS as stabilizing agents for the separation of sulphatestabilized polystyrene (SSPS) nanospheres by AGE and light-scattering video tracking detection. They measured how the agarose gel concentration, stabilizing agent type and concentration and electric field strength affected the electrophoretic mobility of SSPS NPs with radii ranging up to about $150 \mathrm{~nm}$. The same authors [16] introduced and adaptive deconvolution method applying passivated gel electrophoresis to resolve size distributions of multi-modal mixtures of several size standards and a polydisperse nanoemulsion of SSPS.

AGE has been extensively used to characterize pure or synthetic NPs mixture and to confirm the exit of NPs functionalization. However, until our knowledge, there are not publications regarding the use of AGE for NPs sizes characterization in industrial or consumer products. The main difficulties of characterizing complex samples are that NPs are not homogeneous, concentrations can be very low, and matrices can be very different from standards. The object of this work is to evaluate the use of AGE for characterization of AgNPs in an industrial product. We studied the behaviour of different sizes AgNPs standards and an industrial product containing AgNPs with a casein matrix (Collargol) under different electrophoretic conditions. Citrate capped-metallic nanoparticles are the most widely employed, hence they were selected as commercial standards in this study. Influence of different stabilizing agents, surfactants, 
buffers and functionalizing agents has been investigated for the characterization of AgNPs in the industrial product by AGE. In addition possibilities of AGE for protein corona characterization were also demostrated. Laser Ablation-ICP-MS (LA-ICP-MS) has been used increasingly for metalloproteins analysis after separation by PAGE [17]. In our case LA-ICPMS has been used for detection of AgNPs after separation by AGE to confirm visual results obtained in gels.

\section{Materials and methods}

\subsection{Instrumentation}

AGE was carried out with a Sub-Cell ${ }^{\circledR}$ GT horizontal electrophoresis cell and PAGE was carried out with MiniProtean ${ }^{\circledR} 3$ electrophoresis cell. Both cells were connected to a PowerPac ${ }^{\mathrm{TM}}$ basic power supply. For LA-ICP-MS measurements, the gels were vacuum dried with a model 583 gel dryer. All the electrophoretic instrumentation was from Bio-Rad Laboratories (Hercules, USA). Gel images were taken with a consumer digital camera (Canon (PowerShot A2400 IS).

UV-Vis data were obtained using a 8452A Diode Array Spectrophotometer (Hewlett Packard, Palo alto, CA, USA).

A Nd-YAG LA system operating at 213 nm (UP-213, New Wave Research, Huntington, UK) was coupled to an ICP ion source mass spectrometer (Perkin Elmer Elan DRC-e, Toronto, Canada). Prior to all experiments, the ICP-MS instrument was optimized for routine multielemental analysis following the manufacturer's instructions. The LA-ICP-MS set-up was optimized as described elsewhere [18]. Typical operating parameters for LA-ICP-MS measurements are summarized in Table 1s (supporting info). For LA-ICP-MS measurements, each electrophoretic unstained gel lane was completely and continuously scanned by the laser beam (at a scan translation velocity of $60 \mu \mathrm{m} \mathrm{s}^{-1}$ ). The dried gels were cut in two pieces of a size to fit in the ablation chamber. The gel pieces were glued to the support using double-sides adhesive tape. The ablated material was transported by Ar as carrier gas into the ICP. The isotopes monitored were ${ }^{107} \mathrm{Ag}$ and ${ }^{109} \mathrm{Ag}$ and ${ }^{13} \mathrm{C} .{ }^{13} \mathrm{C}$ was used as an internal standard for 
correcting signal drift. Time dependent current is measured during translation of laser scan. The measured current time profile data of the ICP-MS was exported to Excell software, where current time profile can be calculated by transforming time into a length $(\mathrm{cm})$ scale (using the selected translation velocity).

\subsection{Reagents, standards and samples}

Tris, SDS, glycine, Certified ${ }^{\mathrm{TM}}$ Megabase Agarose, EDTA, ammonium persulfate, TEMED, 30\% 29:1 acrylamide/biscrylamide solution (3.3\% C), quick start Bradford protein assay and Coomassie $^{\circledR}$ brilliant blue G-250 of electrophoresis purity were purchased from Bio-Rad Laboratories (Hercules, CA, USA). 2-Mercaptoethanol, was provided by Fluka BioChemika (Buchs, Switzerland).

Mercaptosuccinic acid (TMA), sodium dodecylbenzenesulfonate (SDBS), PEG (molecular weight (MW) (g/mol): 1500), BSA (MW (kDa): 67), human transferrin (MW (kDa): 76.5), SigmaMarker ${ }^{\mathrm{TM}}$ wide range, 6500-200000 Da (as MW standard for SDS-PAGE) and glycerol were supplied by Sigma-Aldrich Chemie (Stenheim, Germany).

Boric acid crystallized (molecular biology grade) was from Panreac (Barcelona, Spain) and $\mathrm{HNO}_{3}(69-70 \% \mathrm{v} / \mathrm{v}$ ) (for trace metal analysis), was purchased from J. T. Baker (Phillipsburg, New York).

Ultrapure water (18.2 M $\left.\Omega \mathrm{cm}^{-1}\right)$ was obtained from a Milli-Q system (Millipore, Bedford, MA, USA).

A series of suspensions of Ag NPs (stabilized by citrate with a net negative surface charge) of different diameters [10 nm, $100 \mathrm{mg} \mathrm{L}^{-1}$ (PlasmaChem, Berlin, Germany), 20 $\pm 5 \mathrm{~nm}$, and $40 \pm 5$ nm (20 mg L ${ }^{-1}$, NanoComposix, San Diego, USA)] were used as commercial standards (Sts). The industrial product is named Collargol, CAS registry number 9007-35-6. It is described in pharmacopeias as a strong antiseptic. This product (granulated powder, silver nanoparticles in a protein (casein) matrix, silver content $68 \pm 1 \%$, average diameter ca. $15 \mathrm{~nm}$ ) was provided by the manufacturer (Laboratorios Argenol, Spain) and was used in solution. Fifty milligrams of 
this product was suspended in $50 \mathrm{~mL}$ of ultrapure water. This product has been characterized by our group in a previous publication [19].

\subsection{Agarose Gel Electrophoresis}

The preparation of agarose gel $(0.5-2 \%)$ is described in supporting info. The initial electrophoresis buffer (EB) was 0.5 x TBE, mostly used in bibliography. Modifications of this initial EB including use of Tris Glycine, different stabilizing agents (PEG, SDS and SDBS) and functionalizing agents (TMA and proteins), were evaluated in order to study the behaviour of AgNPs standards (stabilized with citrate) and the industrial product (Collargol). The different AgNPs were mixed with loading buffer (LB) (ratio 1:1) containing similar composition to the several EB used and glycerol $(30 \% \mathrm{~m} / \mathrm{m})$. Gel compositions, EB and LB used in different experiments are listed in table 1. After mixing AgNPs with LB, they were loaded into the wells (sample volume per well: $15 \mu \mathrm{L}$ ). The electrophoresis cell was connected to the power source and run during $1 \mathrm{~h}$ at a constant voltage of $150 \mathrm{~V}$, corresponding to a potential gradient of $10 \mathrm{~V}$ $\mathrm{cm}^{-1}$.

\section{Results and discussion}

\subsection{Use of stabilizing agents}

Initial studies (experiment 1 in table 1) were made with different sizes (10, 20 and $40 \mathrm{~nm}$ ) AgNPs standards applying electrophoretic conditions mostly used in bibliography $(0.5-2 \%$ agarose and $0.5 \mathrm{x}$ TBE as electrophoresis and loading buffer). For the different agarose concentrations investigated and the different sizes standards, it was observed (figure no shown) that the signal corresponding to both Ag isotopes appears in the beginning of gel lane (just in the well). The reason of this is because AgNPs did not move into the gel either due to the loss of weakly bound citrate ligands or interaction of NPs with the gel. The same behaviour was observed for the industrial product (Collargol) (figures no shown). This fact had been already described for citrate capped Au NPs [14]. Crew et al. observed that the unmodified Au NPs (with citrate capping) basically stayed in the well of the gel whereas the miRNA-conjugated AuNPs travelled to a certain distance in the gel. Zeta potential value for Collargol has been determined in the $2-11 \mathrm{pH}$ range, in a previous publication [19], resulting a zeta potential 
around $-(30-35) \mathrm{mV}$ at $\mathrm{pH} 8.3$. Taking into account zeta potential values of Collargol the NPs should move in the gel according to their size/charge ratio (s/z) but this was not observed. Then we tried to optimize electrophoretic conditions to get AgNPs Sts and sample moving in the gel according to $\mathrm{s} / \mathrm{z}$.

Zhu and Mason [15] proposed the use of passivation agents (PEG and SDS) in gel for separation of SSPS NPs by AGE. They used $0.195 \%$ agarose gel and preferred the use of PEG as passivation agent because is neutral and then it cannot affect the charge on NPs and therefore their electrophoretic mobility and velocity. In our experiment 2 (see table 1) we used a gel containing $0.5 \%$ agarose, $1 \mathrm{mM} \mathrm{PEG} \mathrm{(MW} 1500 \mathrm{~g} / \mathrm{mol}$ ) and $0.5 \mathrm{x}$ TBE. Similar results as the ones obtained in experiment 1 without PEG were obtained. Both AgNPs St and sample stayed in the well of the gel.

In next experiment (experiment 3) SDS was used as stabilizing agent. SDS has been extensively used for metal NPs characterization by CE. Liu et al. [20] demonstrated that SDS is a good stabilizing additive for the characterization of AgNPs' sizes during their separation by CE. SDS added in the running electrolyte prevents AgNPs from coalescing, protecting the suspended AgNPs so that they have longer lifetimes relative to the suspensions in sodium citrate. In a most recent publication Franze and Engelhard [21] developed a method based on the use of MEKC coupled to ICP-MS for characterization and speciation of Au and Ag NPs. They applied the method for the speciation analysis of a dietary supplement. Using Ag and Au commercial Sts with different nominal diameter $(5,20$ and $50 \mathrm{~nm})$, they found $60 \mathrm{mM}$ as optimum SDS concentration As the use of SDS has been successfully using CE, in our work we tried SDS concentrations in the range of $0.1-2 \%(60 \mathrm{mM}$ is equivalent to $2 \%)$. We added SDS either in the gel, electrophoresis buffer and loading buffer (see table 1). Again for the different SDS concentrations studied AgNPs Sts. did not move in the gel and stayed in the well or in the first part of the gel. However, the behaviour for the industrial product was completely different. In Figure 1a-d electropherograms of Collargol after AGE and LA-ICP-MS detection are presented with increasing SDS concentrations. The advance of NPs in the gel clearly improves adding SDS to gel, EB and LB. Similar electropherogram as the one shown in figure 1d was obtained 
for $2 \%$ SDS but $1 \%$ SDS was chosen as optimum concentration in gel and EB to avoid problems with potential variations observed when 2\% SDS was used in EB. The different behaviour of AgNPs Sts and the industrial product in the presence of SDS is due to the different composition of both standards and sample. As described above, Collargol contains silver nanoparticles in a protein (casein) matrix whereas standards are stabilized with citrate with a more labile binding to NPs.

Qu el al. [22] proposed the use of SDBS better than SDS to improve resolution when the size difference of the adjacent NP fraction was less than $10 \mathrm{~nm}$ in a separation of a AuNPs commercial standards mixture $(5,15,20$ and $30 \mathrm{~nm})$ by CE coupled to ICP-MS. Due to the higher hydrophobicity of SDBS the self-assembly of SDBS enabled the generation of a surfactant layer with larger charge differences between different particle sizes, resulting in better separation. Therefore we thought to use SDBS to get AgNPs St moving into the agarose gel and 1\% SDBS (similar SDBS concentration as the optimum used in [22]) was added in gel, EB and LB (see experiment 4 in table 1). No better results than using SDS were obtained. For the industrial product, a band was obtained in the second half of the gel (similar to the one obtained in figure 1d) but AgNPs Sts did not move in the gel according to their s/z either.

\subsection{Use of Mercaptosuccinic acid (TMA) as functionalizing agent}

As no better results were obtained with SDBS as surfactant, 1\% SDS was used in gel and EB and 2\% SDS in LD for next experiments. In addition, the use of borate and EDTA was avoided to avoid NPs agglomeration. A similar electrophoresis buffer as the one used in LAEMLI method for PAGE method [23] was investigated in experiment 5 (see table 1). No improvement was obtained for different sizes AgNPs Sts either but for the industrial product a narrower band (Figure 1s in supporting info) than the one obtained using TBE (figure 1d) was observed in the same gel position. Then Tris Glycine was used instead of $0.5 x T B E$ for next experiments. TMA has been described as stabilizer of citrate capped AuNPs previously to characterization by on-line GE and ICP-MS detection [24]. The introduction of carboxylate groups makes AuNPs bear negative charges at basic $\mathrm{pHs}$ and furthermore, carboxylate groups substantially improves the stability of NPs through electrostatic repulsion due to disulfide ring with gold atoms. 
Lopez-Lorente et al. [25] found that adding TMA into the electrophoretic buffer in an Ag and Au NPs separation by CE, migration of AgNPs became slower than AuNPs as a consequence of the highest affinity of TMA for silver atoms that selectively induced to AgNP functionalization. In experiment 6 (see table 1), we added 0.1\% TMA (similar concentration as the one used in reference [25]) into gel, EB and LB. In figure 2, a photograph of gel obtained is presented (an extended view of bands obtained is shown). By contrast to our previous experiments described above, movement of 10, 20 and $40 \mathrm{~nm}$-AgNPs commercial Sts (lanes 3, 4, 5 and 7, 8 and 9 in gel) is observed appearing in the last part of the gel. $40 \mathrm{~nm}$-AgNPs St moves more than $20 \mathrm{~nm}$ AgNPs St and this more than $10 \mathrm{~nm}-\mathrm{AgNPs}$ St. This fact can be explained because according to Helfrich and Bettmer [24] the ratio between the number of core atoms/ions to the number of ligand molecules (TMA) raises with increasing NP size. Then $40 \mathrm{~nm}-\mathrm{Ag}$ NPs St will have more negative charge than $20 \mathrm{~nm}$-Ag NPs St and $10 \mathrm{~nm}$-AgNPs St respectively. The s/z will be higher for $40 \mathrm{~nm}-\mathrm{Ag}$ NPs St than for $20 \mathrm{~nm}-\mathrm{Ag}$ NPs St and so on, which makes $40 \mathrm{~nm}-\mathrm{Ag}$ NPs St moves faster. The apparent electrophoretic mobility, $\mathrm{m}_{\text {app }}\left(\mathrm{cm}^{2} \mathrm{~V}^{-1} \mathrm{~s}^{-1}\right)$ has been determined by the equation of $m_{\text {app }}=\left(\mathrm{L}_{\text {tot }} \mathrm{L}_{\text {eff }}\right) /\left(\mathrm{V}_{\text {sep }} \mathrm{t}_{\mathrm{sep}}\right)$ where $\mathrm{L}_{\text {tot }}$ is the total length of the separation medium (distance between two electrodes in the applied apparatus), $\mathrm{L}_{\text {eff }}$ is the migrated distance of the each different $\mathrm{NP}, \mathrm{t}_{\text {sep }}$ is the time of separation and $\mathrm{V}_{\text {sep }}$ is the applied voltage of the system [26]. Although there is a linear relationship between $\mathrm{m}_{\text {app }}$ and the NPs diameter (d) in nanometer $(\mathrm{y}=3 \mathrm{E}-07 \mathrm{~d}+0,0003, \mathrm{r}=0.997)$, when diameter of AgNPs in sample is calculated from this equation, a diameter of $9.6 \mathrm{~nm}(\mathrm{RSD}=1.21 \%)$ is obtained. This value is much lower than the sample average diameter $(15 \mathrm{~nm})$ characterized in a previous work [19]. Due to the different composition of the industrial product (AgNPs in a casein matrix) the functionalization with TMA is different from the functionalization of AgNPs commercial Sts (citrate capped). Then if the charge for standards and sample is different, the NPs size characterization by AGE based on the size/charge ratio is not possible.

After the study of influence of different stabilizing agents, buffers and functionalizing agents, unsuccessful characterization of AgNPs in the industrial product using commercial standards is concluded. 


\subsection{Use of $A G E$ for protein corona characterization}

The interaction of inorganic NPs with biomolecules forming the so-called protein corona (PC) has attracted increasing attention as demonstrated by the great number of publications [27, 28]. 1D- and 2D PAGE are the gel electrophoretic techniques mostly used for characterization of PC [29]. Until our knowledge AGE has been only applied to study the evolution from discrete conjugates to PC between BSA and AgNPs [30]. We have investigated the use of AGE for characterizing the PC formed between Collargol sample and BSA and Transferrin (TF). After incubation of sample with both proteins (see incubation procedure in supporting info), PC formation was confirmed by UV-Vis Spectroscopy and SDS-PAGE. There is a red shift (to higher $\lambda$ values) of the characteristic peak of superficial plasmon resonance (SPR) for AgNPs at $405 \mathrm{~nm}$ in the presence of both proteins (UV-Vis spectra in figure $2 \mathrm{~s}$ and data in table $2 \mathrm{~s}$ of supporting info) which confirms the PC formation [31]. The protein content in the supernatant after first centrifugation was quantified by Bradford assay. The percentage of protein in the supernatants relative to the control sample without NPs (proteins without Collargol) was calculated and the percentage of protein forming PC (calculated as difference between initial protein concentration minus protein concentration in supernatant) was $39.8 \pm 0.83 \%$ and $46.7 \pm$ $1.33 \%$ for BSA and TF respectively. The higher $\%$ of TF forming PC compared to BSA confirms the higher red shift of $\lambda$ obtained in UV-Vis spectra. The PC formation was also confirmed by SDS-PAGE. Pellets obtained after 1 and 3 washes with ultrapure water were analyzed according to Laemmli method [23] with stacking gel prepared at $4 \%$, and resolving gel at $10 \%$. The gel obtained (figure $3 \mathrm{~s}$ of supporting info) shows both proteins (BSA and TF) forming PC with AgNPs.

Pellets obtained after 3 washes with ultrapure water were also analyzed by AGE method using electrophoretic conditions of experiment 5 (see table 1). The advantage of using AGE instead of SDS-PAGE is that AGE is a non-denaturing method (compared with SDS-PAGE) with pore size enough to enable the separation of PC without breaking binding between NPs and proteins (opposite to SDS-PAGE). We can confirm the PC formation after AGE separation. The electrophoretic mobilities (in $\left.\mathrm{cm}^{2} \mathrm{~V}^{-1} \mathrm{~s}^{-1}\right)$ of Collargol bound to BSA $\left(3.00 \times 10^{-4}\right)$ and TF 
$\left(2.90 \times 10^{-4}\right)$ are lower than the one of Collargol $\left(3.33 \times 10^{-4}\right)$ (Figure $4 \mathrm{~s}$ and table $3 \mathrm{~s}$ in supporting info). Both PC are delayed in the gel matrix because of their larger hydrodynamic size. In addition PC formed with TF is more delayed than PC formed with BSA due to the higher size of TF (MW: $76.5 \mathrm{kDa}$ ) than BSA, (MW: $67 \mathrm{kDa}$ ). In Figure 3, electropherograms of Collargol (a), Collargol-BSA (b) and Collargol-TF (c) after AGE and LA-ICP-MS detection are presented. Using LA-ICP-MS detection two peaks (which are not visually detected in the gel) are observed in the bands corresponding to Collargol-BSA and Collargol-TF. The double peak can be explained because the excess of proteins concentration (2000 mg L-1) is only two-fold the NP concentration (1000 mg L-1) and then part of NPs remained in the unbound state. This fact was also described by Matczuk et al. [32] who studied the speciation of AuNPs in human serum by CE-ICP-MS. They found that a 20-fold molar excess of albumin about AuNPs led to a deceleration of albumin binding and even after $24 \mathrm{~h}$ of incubation, $5 \%$ of NPs remained in the unbound state. In addition the same authors described a double peak for the interaction of AuNPs with TF due to TF exists in human blood in two forms (iron-free or apo-transferrin and iron-saturated or holo-transferrin).

\section{Concluding remarks}

In this study in and outs of AGE for NPs characterization are described. Although AGE is a successful method to evaluate NPs functionalization or size characterization in pure NMs or synthetic mixtures, when NPs have to be characterized in industrial or consumer products (more complex systems), the different composition of sample and standards has to be taken into account. We have studied the influence of different stabilizing agents (PEG, SDS and SDBS), buffers (TBE and Tris Glycine) and functionalizing agents (TMA and proteins) for the characterization of AgNPs in an industrial product (Collargol, casein-modified AgNPs) by AGE with LA-ICP-MS detection. Citrate capped AgNPs standards were used. Although the use of SDS and Tris Glycine in gel, electrophoresis buffer and loading buffer, allowed observe movement of AgNPs in the industrial product, the different sizes-AgNPs Sts did not move into the gel and stayed in the wells. After functionalization of both sample and standards with TMA, the different sizes-AgNPs standards moved according to their size/charge ratio (obtaining a 
linear relationship between apparent mobility and mean diameter). However the behaviour of the industrial product was completely different from standards leading to a wrong mean diameter calculation (according to bibliography). The reason of this behaviour is that the complete exchange of nitrogen containing protein to thiol is not easily achieved. It is expected that further development using matrix matched NP standards to establish calibration curves for each specific type of NPs will permit the characterization of NPs in real samples. In contrast we demonstrated that AGE with LA-ICP-MS detection is an alternative method to confirm the protein corona formation between the industrial product and two proteins (Bovine Serum Albumin and transferrin) maintaining NPs-protein binding (what is not possible using SDSPAGE). 


\section{Acknowledgements}

This work was sponsored by the by Spanish Ministry of Science and Technology, project no CTQ 2012-38091-CO2-00. The authors also thank Laboratorios Argenol S.L. for providing Collargol sample and Cristina Villanueva for the UV-Vis and SDS-PAGE data regarding protein corona studies. LA-ICP-MS measurements were performed in the facilities of the Servicio General de Apoyo a la Investigación-SAI, Universidad de Zaragoza.

\section{Conflict of interest statement}

There are no financial/commercial conflicts of interest. 


\section{References}

[1] Surugau, N., Urban, P.L., J. Sep. Sci., 2009, 32, 1889-1906.

[2] López-Lorente, A.I., Simonet, B.M., Valcarcel, M., Trends in Analytical Chemistry, 2011, $30,58-71$.

[3] Fedotov, P.S., Vanifatova, N.G., Shkinev, V.M., Spivakov, B.Y., Anal. Bioanal. Chem., $2011,400,1787-1804$

[4] Chung-Shu, W., Fu-Ken, L., Fu-Hsiang, K., Anal. Bioanal. Chem., 2011, 399, 103-118.

[5] Jing-fu, L., Su-juan, Y., Yong-guang, Y., Jing-bo, Ch., Trends in Analytical Chemistry, 2012, $33,95-99$.

[6] Hanauer, M., Pierrat, S., Zins, I., Lotz, A., Sönnichsen, C., Nano Letters, 2007, 7, 2881-2885.

[7] Hames, B.D., Gel Electrophoresis of Proteins : A practical approach (Third Edition), Oxford University Press, Oxford, 1998.

[8] Rickwood D. and Hames B.D., Gel Electrophoresis of Nucleic Acids A Practical Approach (Second Edition), IRL Press, at Oxford University Press, Oxford, 1990.

[9] Wang, S., Mamedova, N., Kotov, N.A., Chen, W., Studer, J., Nano Letters, 2002, 2, 817-822.

[10] Krizkova, S., Dostalova, S., Michalek, P., Nejdl, L., Kominkova, M., Milosavljevic, V., Moulick, A., Vaculovicova, M., Kopel, P., Adam, V., Kizek, R., Chromatographia, 2015, 78, 785-793.

[11] Wu, W., Huang, J., Wu, L., Sun, D., Lin, L., Zhou, Y., Wang, H., Li, Q., Separation and Purification Technology, 2013, 106, 117-122.

[12] Bartczak, D., Kanaras A.G. et al, Langmuir, 2011, 27, 10119-10123.

[13] Beskorovaynyy, A.V., Kopitsyn, D.S., Novikov, A.A., Ziangirova, M., Skorikova, G.S., Kotelev, M.S., Gushchin, P.A., Ivanov, E.V., Getmansky, M.D., Itzkan, I., Muradov, A.V., Vinokurov, V.A., Perelman, L., ACS Nano, 2014, 8, 1449-1456.

[14] Crew, E., Rahman, S., Razzak-Jaffar, A., Mott, D., Kamundi, M., Yu, G., Tchah, N., Lee, J., Bellavia, M., Zhong, C-J, Anal. Chem., 2012, 84, 26-29.

[15] Zhu, X., Mason, T.G., J. of Colloid and Interface Science, 2014, 428, 199-207.

[16] Zhu, X., Mason, T.G., J. of Colloid and Interface Science, 2014, 435, 67-74. 
[17] Pozebon, D., Scheffler, G.L., Dressler, V.L., Nunes, M.A.G., J. Anal. At. Spectrom., 2014, $29,2204-2228$.

[18] Jimenez, M.S., Rodriguez, L., Bertolín, J.R., Gomez, M.T., Castillo,J.R., Anal. and Bioanal. Chemistry, 2013, 405, 359-568.

[19] Bolea, E., Jiménez-Lamana, J., Laborda, F., Castillo, J.R., Anal. Bioanal. Chem., 2011, 401, $2723-2732$.

[20] Liu, F-K, Ko, F-H., Huang, P-W, Wu, C-H., Chu, T-C, J. of Chromatography A, 2005, $1062,139-145$.

[21] Franze, B., Engelhard, C., Anal. Chem., 2014, 86, 5713-5720.

[22] Qu, H., Mudalige, T.K., Linder, S.W., Anal. Chem., 2014, 86, 11620-11621.

[23] Laemmli, U.K., Nature, 1970, 227, 680-685.

[24] Helfrich, A., Bettmer, J., Intern. J. of Mass Spectrom., 2011, 307, 92-98.

[25] López-Lorente, A.I., Soriano, M.L., Válcarcel, M., Microchim. Acta, 2014, 181, 1789-1796.

[26] Kim, J-Y, Kim, H-B, Jang, D-J, Electrophoresis, 2013, 34, 911-916.

[27] Wolfram, J., Yang, Y., Shen, J., Moten, A.,Chen, Ch., Shen, H., Ferrari, M., Zhao, Y., Colloids and Surfaces B: Biointerfaces , 2014, 124, 17-24.

[28] Lee, Y.K., Choi, E., Webster, T.J., Kim, S., Khang, D., Internat. J. of Nanomedicine, 2015, $10,97-112$.

[29] Capriotti, A.L., Garacciolo, G., Cavaliere, Ch., Colapicchioni, V., Piovesana, S., Pozzi, D., Laganà, A., Chromatographia, 2014, 77, 755-769.

[30] Guo, J., Zhong, R., Li, W., Liu, Y., Bai, Z., Yin, J., Liu, J., Gong, P., Zhao, X., Zhang, F., doi: 10.1016/j.apsusc.2015.09.247.

[31] Ran, C., Poonam, C., Ryan, N., Priyaca, B., Jared, M., Brown, P., Ke, Ch., Applied Physics Letters , 2012, 100, 013703.

[32] Matczuk, M., Anecka, K., Scaletti, F., Messori, L., Keppler, B.K., Timerbaev, A.R., Jarosz, M., Metallomics, 2015, 7, 1364-1370. 


\section{List of figures and captions}

Figure 1. Electropherograms of Collargol with different SDS concentrations in gel, electrophoresis buffer and loading buffer (Experiment 3, Table 1). LA-ICP-MS detection. a) without SDS, b) $0.1 \% \mathrm{SDS}$, c) $0.3 \% \mathrm{SDS}$, d) $1 \% \mathrm{SDS}$ ).

Figure 2. Gel dried after AGE (Conditions of experiment 6, Table 1). 1. Collargol (25 mg L $\left.\mathrm{m}^{-1}\right)$, 2. Collargol (100 mg L$\left.~^{-1}\right), 3.10 \mathrm{~nm}$ AgNP St $\left(20 \mathrm{mg} \mathrm{L}^{-1}\right), 4.20 \mathrm{~nm} \mathrm{AgNP} \mathrm{St}\left(20 \mathrm{mg} \mathrm{L}^{-1}\right), 5.40$ nm AgNP St (20 mg L $\left.{ }^{-1}\right), 6$. Collargol $\left(25 \mathrm{mg} \mathrm{L}^{-1}\right), 7.10 \mathrm{~nm} \mathrm{AgNP} \mathrm{St}\left(20 \mathrm{mg} \mathrm{L}^{-1}\right), 8.20 \mathrm{~nm}$ AgNP St (20 mg L $\left.{ }^{-1}\right), 9.40 \mathrm{~nm}$ AgNP St $\left(20 \mathrm{mg} \mathrm{L}^{-1}\right)$, 10. Collargol (25 mg L $\left.\mathrm{m}^{-1}\right)$, 11. Collargol $\left(1000 \mathrm{mg} \mathrm{L}^{-1}\right)$

Figure 3. Electropherograms of Collargol-proteins complexes. AGE separation conditions: 25 $\mathrm{mM}$ Tris $+1 \%$ SDS in gel, $25 \mathrm{mM}$ Tris $+192 \mathrm{mM}$ glicine $+1 \%$ SDS in electrophoresis buffer, $25 \mathrm{mM}$ Tris $+30 \%$ Glycerol $+2 \%$ SDS in loading buffer (Experiment 5, Table 1). LA-ICP-MS detection. a) Collargol, b) Collargol + BSA, c) Collargol + TF. 
Figure 1.

a)

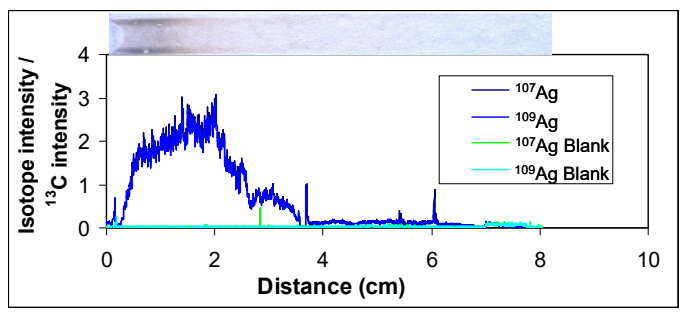

c)

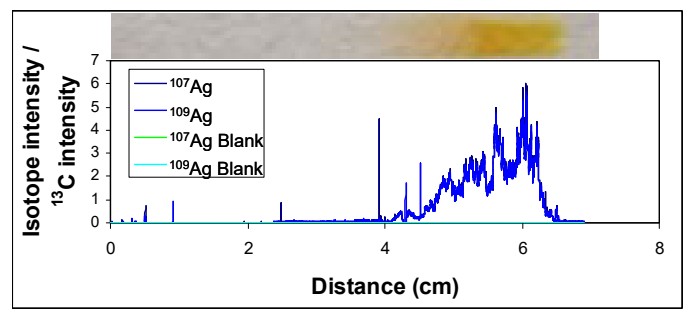

b)

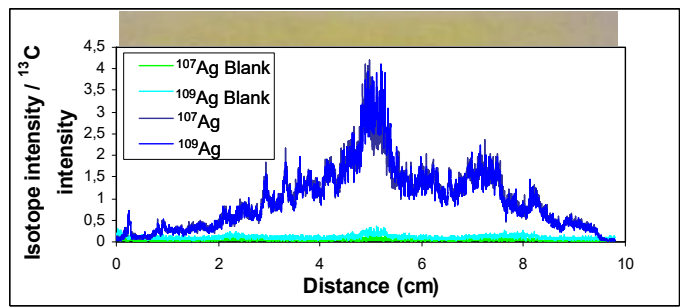

d)

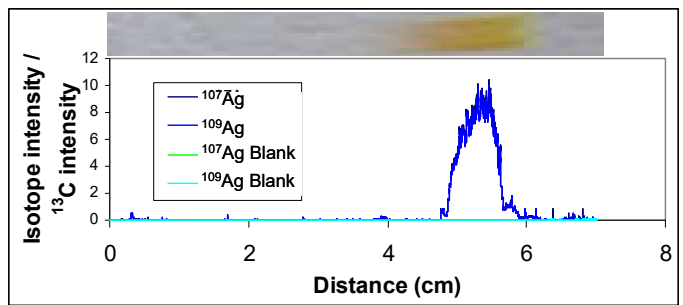


Figure 2

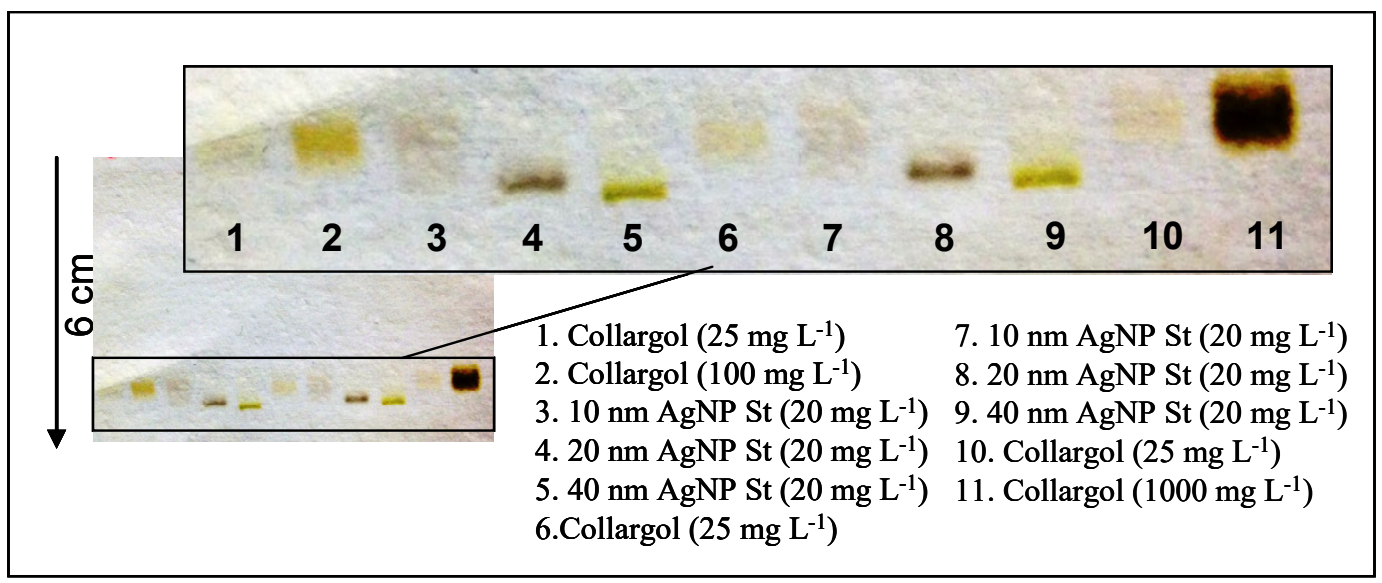


Figure 3

a)

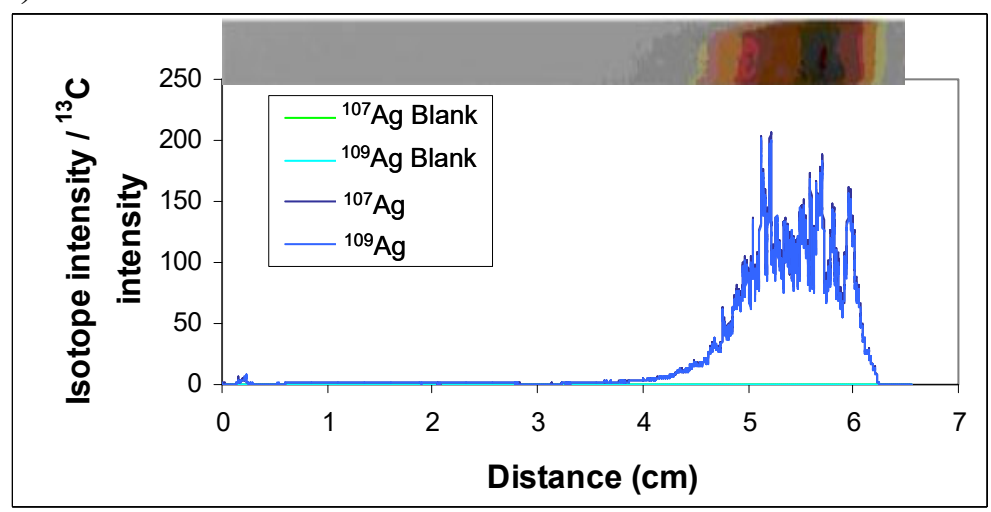

b)

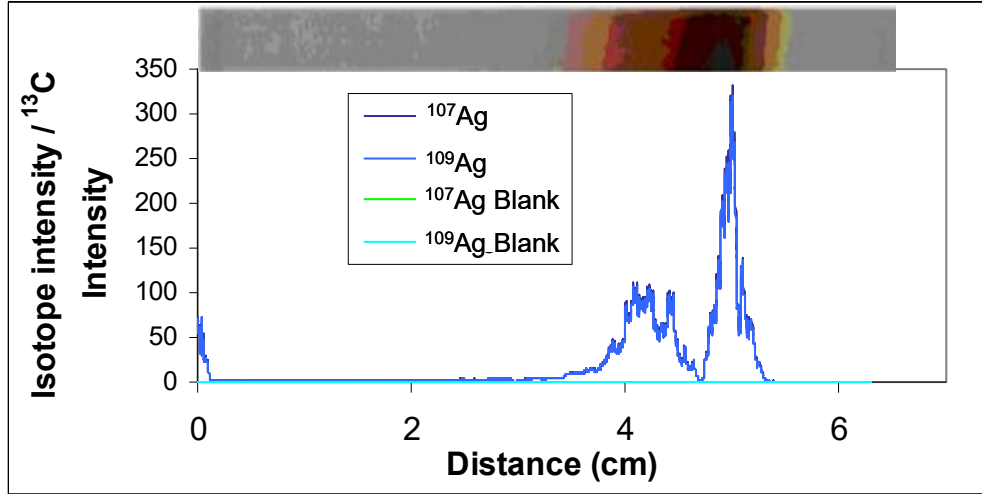

c)

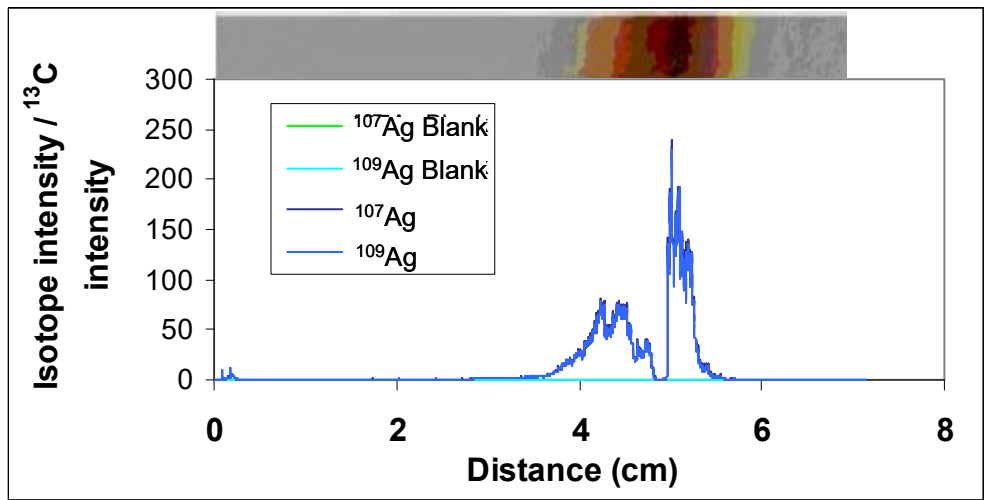


Table 1. Gels, electrophoresis buffers and loading buffers composition (all at $\mathrm{pH} 8.3$ )

\begin{tabular}{|c|c|c|c|}
\hline $\begin{array}{l}\text { Experiment } \\
\text { Number }\end{array}$ & Gel & $\begin{array}{l}\text { Electrophoresis } \\
\text { Buffer (EB) }\end{array}$ & $\begin{array}{c}\text { Loading } \\
\text { Buffer (LB) }\end{array}$ \\
\hline 1 & $0.5-2 \%$ Agarose $+0.5 \times \mathrm{TBE}$ & $0.5 \times \mathrm{TBE}$ & $0.5 \times \mathrm{TBE}+30 \%$ Glycerol \\
\hline 2 & $\begin{array}{c}0.5 \% \text { Agarose }+0.5 \times \mathrm{TBE} \\
+1 \mathrm{mM} \text { PEG }\end{array}$ & $0.5 \times \mathrm{TBE}$ & $0.5 \times \mathrm{TBE}+30 \%$ Glycerol \\
\hline 3 & $\begin{array}{c}0.5 \% \text { Agarose }+0.5 \times \text { TBE } \\
+0.1-2 \% \mathrm{SDS}\end{array}$ & $\begin{aligned} & 0.5 \times \mathrm{TBE} \\
+ & 0.1-2 \% \mathrm{SDS}\end{aligned}$ & $\begin{array}{c}0.5 \times \mathrm{TBE}+30 \% \text { Glycerol } \\
+0.1-2 \% \mathrm{SDS}\end{array}$ \\
\hline 4 & $\begin{array}{c}0.5 \% \text { Agarose }+0.5 \times \mathrm{TBE} \\
+1 \% \mathrm{SDBS}\end{array}$ & $\begin{array}{c}0.5 \times \mathrm{TBE} \\
+1 \% \mathrm{SDBS}\end{array}$ & $\begin{array}{c}0.5 \times \text { TBE }+30 \% \text { Glycerol } \\
+1 \% \text { SDBS SDS }\end{array}$ \\
\hline 5 & $\begin{array}{c}0.5 \% \text { Agarose }+25 \mathrm{mM} \text { Tris } \\
+1 \% \mathrm{SDS}\end{array}$ & $\begin{array}{c}25 \mathrm{mM} \text { Tris }+ \\
192 \mathrm{mM} \text { glycine }+1 \% \text { SDS }\end{array}$ & $\begin{array}{c}25 \mathrm{mM} \text { Tris }+30 \% \text { Glycerol } \\
+2 \% \text { SDS }\end{array}$ \\
\hline 6 & $\begin{array}{c}0.5 \% \text { Agarose }+25 \mathrm{mM} \text { Tris } \\
+1 \% \mathrm{SDS}+0.1 \% \mathrm{TMA}\end{array}$ & $\begin{array}{c}25 \mathrm{mM} \text { Tris }+ \\
192 \mathrm{mM} \text { glycine }+1 \% \text { SDS } \\
+0.1 \% \text { TMA }\end{array}$ & $\begin{array}{c}25 \mathrm{mM} \text { Tris }+30 \% \text { Glycerol } \\
+2 \% \text { SDS }+0.1 \% \text { TMA }\end{array}$ \\
\hline
\end{tabular}

All the experiments were run at constant voltage of $150 \mathrm{~V}$, corresponding to a potential gradient of $10 \mathrm{~V} \mathrm{~cm}^{-1}$ 